\title{
Advancing COVID Vaccination Equity at Federally Qualified Health Centers: a Rapid Qualitative Review
}

J Gen Intern Med 37(4): 1012-3

DOI: $10.1007 / \mathrm{s} 11606-021-07273-8$

(C) Society of General Internal Medicine 2021

\section{INTRODUCTION}

Equitable access to COVID-19 vaccination is crucial to mitigating disproportionate COVID-19 morbidity and mortality in lower-income minority communities in the USA. Federally Qualified Health Centers (FQHCs) are non-profit health systems that receive federal funding to provide primary care to medically underserved communities. FQHCs serve 30 million patients annually, $90 \%$ of whom live below $200 \%$ of the federal poverty line and $63 \%$ of whom are racial/ethnic minorities. ${ }^{1}$ In February 2021, the White House initiated direct allocation of COVID vaccines to FQHCs in a phased program later expanded nationwide. Vaccination appointments at FQHCs were open to the general public. From January to May 2021, over $60 \%$ of vaccinations at FQHCs went to people of color, versus less than $40 \%$ nationally. ${ }^{2}$ The share of vaccinations administered at FQHCs also increased over time, suggesting the important role $\mathrm{FQHCs}$ play in vaccine equity.

This rapid qualitative study documents strategies FQHCs employed to advance COVID vaccine access and build trust in underserved communities. ${ }^{3}$

\section{METHODS}

In March and April 2021, three authors (JTC, RF, CN) conducted semi-structured Zoom interviews with 20 primary care providers (PCPs) and support staff at two FQHCs in two states in different regions of the USA (Table 1). Both FQHCs participated in the initial phase of the federal vaccine program and had existing relationships with the research team from prior studies, facilitating this rapid-response research. Interviewees were selected purposively to include a variety of patient-facing roles, including physicians, nurses, and outreach workers. The interview guide covered COVID vaccination access, equity considerations, communication about vaccination, patient concerns about vaccination, and patient trust. Interviews lasted 30-40 min and were transcribed for analysis. Transcripts were analyzed using an inductive, iterative

Received July 26, 2021

Accepted November 3, 2021

Published online January 3, 2022 approach. The research team developed analytic codes describing themes emerging from the data and piloted codes on a subset of transcripts to develop rules for consistent application. Transcripts were coded by two teams of two researchers, all of whom were trained in qualitative analysis, using NVivo's collaboration cloud (JTC and RF; CN and DP). Coding discrepancies were reconciled by consensus to ensure reliability. This study was granted exemption by the Albany Medical Center IRB.

\section{RESULTS}

Interviewees described operational and provider strategies that facilitated vaccine access and nurtured trust. Long-standing relationships with patients and communities proved key to FQHC success (Table 2).

Barriers to vaccination included complicated Internet-based appointment booking systems, lack of transportation, time constraints due to work and childcare, and unanswered questions about vaccines. FQHCs reduced these barriers by offering phone and in-person appointment booking; reserving vaccination appointments for highest-risk populations; providing transportation assistance and pop-up/mobile vaccine clinics in convenient locations; and offering weekend appointments. FQHCs also leveraged existing patient-provider and community relationships to share information and nurture trust. These actions included responding individually to patient vaccination concerns; sharing providers' own vaccination experiences with hesitant patients; partnering with churches and community organizations; outreach to farms and other workplaces; and continuing to help patients with needs beyond health care. Interviewees viewed these actions as integral to demonstrating trustworthiness.

Table 1 Study Participants

\begin{tabular}{llll}
\hline \hline & New York & Tennessee & Total \\
\hline Role & & & \\
$\quad$ Primary care provider & 8 & 6 & 14 \\
Other Health Center Staff & 2 & 4 & 6 \\
Gender & 8 & 10 & 18 \\
Female & 2 & 0 & 2 \\
Male & 3 & & \\
Race/ethnicity & 1 & 1 & 4 \\
Asian or South Asian & 2 & 3 & 5 \\
Black & 4 & 5 & 9 \\
Latinx & & & 2 \\
White & & & \\
\hline
\end{tabular}


Table 2 FQHC Actions to Mitigate Barriers and Build Trust

\begin{tabular}{l}
\hline \hline FQHC actions \\
\hline $\begin{array}{l}\text { Reserving appointments } \\
\text { special populations }\end{array}$ \\
$\begin{array}{l}\text { Non-Internet options for } \\
\text { scheduling appointments }\end{array}$
\end{tabular}

scheduling appointments

Mobile vaccination sites

Partnering with churches and community organizations

Outreach to farms and other workplaces

Personalized responses to patient concerns about vaccination

Sharing provider vaccination experiences with hesitant patients

Helping patients with needs beyond health care

Trust building

Quotes from interviews

"We will block in the schedule a certain number of vaccine slots and say, 'We're saving these for the most high-risk people who have difficulty accessing." (Participant 7, Tennessee) "If you come to the clinic and you ask about [vaccination], we have staff who will just sign you up and put you in the queue. What we did not want is the lack of digital literacy or digital health access to be a factor." (Participant 2, Tennessee)

"We're planning some events now, with other individuals and agencies that serve people who don't have homes, to do vaccinations with our mobile clinic. Literally just going to the places where they're more likely to sleep, and vaccinating." (Participant 3, Tennessee)

"We called one of our community stakeholders and she's a lady that's very connected to her church, and she brought her whole congregation, and everybody got vaccinated."

(Participant 5, Tennessee)

"We actually go out [to farms]. . . and speak to them about the vaccines and Covid. And not just farm workers, but we also have daycares and other community centers that we go to... and then have them reach out to us and let us know what their thoughts are."

(Participant 18, New York)

"My next question always is before I open my mouth is like, "what's their concern about it?' And once I hear that concern, I could target my and tailor my answer accordingly... So I always ask them what their concern is when they say 'no', and then go from there.' (Participant 12, New York)

"I tell them, 'Hey, you know, I've been vaccinated, I do have some medical conditions.' So, you know, it is scary, but it's better they get vaccinated.

(Participant 11, Tennessee)

"It's not just medical care, it's everything and anything that they may need, they can reach out to us. So I think that's allowed them to have a lot of trust, and we kind of work like a family." (Participant 18, New York) "Poor people of color have a have a daily lifelong experience of not being respected by people in institutions and not having legitimate access to institutions. And so I think it takes some time to demonstrate that, in fact, one respects, one is respected. And once that happens, then there begins to be a foundation of trust. But it takes time." (Participant 15, New York)

\section{DISCUSSION}

Vaccination strategies are crucial to building community immunity against COVID-19 and mitigating its disproportionate impacts on lower-income communities of color. Access barriers and

distrust present persistent challenges to national vaccination efforts. FQHCs have responded to these challenges with strategies that build on and strengthen existing community ties and patientprovider relationships. These strategies facilitated vaccine access by helping people make and keep appointments and by providing information in a context of trustworthiness. ${ }^{4}$

Notably, FQHCs initiated vaccine clinics and outreach as part of their public health mission. Vaccine efforts served the general public, and relied on volunteer staff until federal support was forthcoming. As one interviewee noted, "in a country that doesn't have a coherent health care system, we stepped in as community health centers to say, 'we will play the role of the public health system." Their greater success at vaccinating people of color holds lessons for other PCPs, such as medical practices and outpatient clinics. FQHC strategies could serve as a national blueprint for how to support just vaccine allocation and improve health equity. Going forward, community-based vaccination strategies should prioritize FQHCs and other PCPs, and include opportunities to learn from $\mathrm{FQHC}$ approaches.

Johanna T. Crane, Ph.D. ${ }^{1}$

Danielle Pacia, M.B.E. ${ }^{2}$

Rachel Fabi, Ph.D. ${ }^{3}$

Carolyn Neuhaus, Ph.D. ${ }^{2}$

Nancy Berlinger, Ph.D. ${ }^{2}$

${ }^{1}$ Alden March Bioethics Institute, Albany Medical College,

Albany, NY, USA

${ }^{2}$ The Hastings Center,

Garrison, NY, USA

${ }^{3}$ SUNY Upstate Medical University, Syracuse, NY, USA

Corresponding Author: Johanna T. Crane, Ph.D.; Alden March Bioethics Institute, Albany Medical College, Albany, NY, USA (e-mail: cranej1@amc.edu).

\section{REFERENCES}

1. Health Resources \& Services Administration. Ensuring Equity in COVID19 Vaccine Distribution. https://www.hrsa.gov/coronavirus/health-center-program. Accessed 29 June 2021.

2. Corallo B, Ärtiga S, Tolbert J. Are Health Centers Facilitating Equitable Access to COVID-19 Vaccinations? A June 2021 Update. Kaiser Family Foundation. https://www.kff.org/coronavirus-covid-19/issue-brief/arehealth-centers-facilitating-equitable-access-to-covid-19-vaccinations-ajune-2021-update/. Accessed 29 June 2021.

3. Vindrola-Padros C, Chisnall G, Cooper S, et al. Carrying Out Rapid Qualitative Research During a Pandemic: Emerging Lessons From COVID19. Qual Health Res. 2020;30(14):2192-2204.

4. Warren RC, Forrow L, Hodge DA, Truog, RD. Trustworthiness before Trust - Covid-19 Vaccine Trials and the Black Community. N Engl J Med 2020; 383:e121.

Publisher's Note: Springer Nature remains neutral with regard to jurisdictional claims in published maps and institutional affiliations. 